vantage is especially marked when applied to substances boiling at high temperatures.

5 . The thermodynamical equations relating heats of vaporization, of sublimation and of fusion and their relation to the vapor pressure and sublimation pressure are briefly reviewed and empirical methods for calculating each from the freezing and boiling points for normal liquids are proposed.

BLOOMINGTON, ILLINOIS

\title{
INVESTIGATION BY MEANS OF THE HYDROGEN ELECTRODE OF THE CHEMICAL REACTIONS INVOLVED IN WATER PURIFICATION ${ }^{1}$
}

By R. E. GREENFIEID WITH A. M. Buswei.

Received February 13, 1922

Although titration of calcium and magnesium carbonates and precipitation of calcium carbonate, magnesium and aluminum hydroxides are common chemical reactions encountered in one way or another in a variety of instances, few definite or direct data are available concerning the course of these reactions. The titration of the alkalinity of calcium carbonate or magnesium carbonate solutions assumes that the end-points are the same as those obtained when titrating sodium carbonate solutions. Little is known concerning the velocities of the precipitation reactions and the optimum conditions for complete precipitation.

The use of the hydrogen electrode for following the courses of these reactions seemed to promise a method for obtaining more exact data both as to the end-point in alkalinity titrations and with respect to conditions for the precipitations. The reactions thus studied were: (1) the titrations of calcium carbonate and magnesium carbonate with a strong acid; (2) the precipitation of calcium carbonate (a) by the addition of hydroxide to the bicarbonate (b) by the addition of carbonate to calcium chloride; (3) the precipitation of magnesium hydroxide (a) from the carbonate (b) from the sulfate; (4) the precipitation of aluminum hydroxide.

Hildebrand ${ }^{2}$ had titrated sodium carbonate with a strong acid using the hydrogen electrode but had not titrated calcium carbonate or magnesium carbonate. He aiso followed the precipitation of aluminum hydroxide with the same device. The apparatus used was, however, much less sensitive than that now available. The concentrations of his solutions were not given by Hildebrand but they were presumably $0.1 \mathrm{~N}$ or more concentrated. The concentrations dealt with in water analysis and purification are so very low that it seemed advisable to repeat his work using more dilute solutions and a sensitive apparatus.

${ }^{1}$ Abstract of thesis submitted by R. E. Greenfield in partial fulfilment of the requirements for the degree of Doctor of Philosophy, at the University of Illinois, August, 1921.

${ }^{2}$ Hildebrand, This JourNaL, 35, 847-71 (1913). 
Blum ${ }^{3}$ published hydrogen electrode titration curves for the precipitation of aluminum hydroxide which were similar to those of Hildebrand. He also determined the optimum limits of hydrogen-ion concentration for the precipitation of aluminum hydroxide in rock analysis. These values were obtained by adjusting the reaction of his solution to a certain value of the hydrogen-ion concentration and then boiling. When less than $0.1 \mathrm{mg}$. was found in $100 \mathrm{cc}$. of the supernatant liquid after boiling, the precipitation was considered complete. While furnishing valuable data for analytical procedure Blum's paper unfortunately does not enable us to decide upon the best conditions for precipitation of aluminum hydroxide in the cold.

The other reactions studied have not, as far as we can learn, been investigated with reference to hydrogen-ion changes during their course.

Apparatus.- The set up consisted of Leeds and Northrup Type $\mathrm{K}$ potentiometer; a No. 2500 galvanometer with a sensitivity of 396 megohms, a period of 3.4 seconds and a coil resistance of 1800 ohms; an Eppley cadmium cell certified by the U. S. Bureau of Standards, and a satirated calomel half-element, Fales ${ }^{4}$ type.

The chemicals for the calomel half-cells were prepared by the usual method as described by Fales, 4,5 Clark, ${ }^{\circ}$ et al.

The water used in the preparation of all solutions was prepared by redistillation of the ordinary grade of distilled water after the addition of alkaline permanganate, the distillate being rejected until it failed to give a test for ammonia with Nessler's reagent. The water so prepared was stored in Pyrex flasks. All chemicals used other than the ones especially taken up in other places were C. P. of the highest grade commercially obtainable. The sodium hydroxide was prepared by dilution of a clear saturated solution with water free from carbon dioxide, standardized with benzoic acid obtained from the Bureau of Standards and fused immediately before using. The hydrochloric acid solution was standardized from the sodium hydroxide solution. All volumetric ware used had been certified by the U.S. Bureau of Standards. The weights used were carefully checked against a set calibrated by the Bureau of Standards.

In calculating the hydrogen-ion concentrations which we have expressed in Sörensen's $P_{H}$ numbers, the following formula has been used.

$$
\frac{E_{\text {obs. }}+E_{\text {bar. }}-E_{\text {calc. }}}{0.00019837 T}=P_{\text {H }}
$$

in which $E_{\text {obs. }}$ is the observed over-all voltage; $E_{\text {bar. }}$ is the correction for barometric pressure and $E_{\text {calc. }}$ is the voltage of the saturated calomel electrode referred to the normal hydrogen electrode as zero and was taken as 0.2466 volts at $25^{\circ 5,7}$. $T$ is absolute temperature.

\section{The Titration of Carbonate Solutions}

One of the most common methods of estimating the normal carbonate content of a solution is to "titrate with a standard acid until the solution

3 Blum, This Journal, 38, 1280 (1916).

4 Fales and Vosburgh, ibid., 40, 1293 (1918).

' Fales and Mudge, ibid., 42, 2434 (1920).

"Clark, "The Determination of Hydrogen Ion Concentration," Williams and Wilkins, Baltimore, 1911.

7 Ellis, ibid., 38, 737 (1916). 
is neutral to phenolphthalein," and in the same manner the total carbonate and bicarbonate content may be estimated if methyl orange is used in place of the phenolphthalein.

If the hydrogen-ion concentration be determined at various steps in the titration and these determinations be plotted as the abscissa against the quantity of acid used a curve is obtained which shows two inflection points, the first inflection occurring when acid equivalent to $1 / 2$ the normal carbonate had been added and the second at the point where acid equivalent to all the carbonate had been added. These curves are useful in confirming the suitability of the indicators used, since in order that the determination should be of the highest accuracy the indicator used should show its end-point color at a hydrogen-ion concentration corresponding to that of the inflection point of the curve. Such data as have already been published show that the above mentioned indicators are about as satisfactory for the purpose as could be expected.

In order to confirm these findings more fully data were obtained for the titrations of $0.02 \mathrm{~N}, 0.01 \mathrm{~N}$ sodium carbonate, $0.01,0.005,0.002 \mathrm{~N}$ calcium bicarbonate and $0.01 \mathrm{~N}$ magnesium bicarbonate solutions.

A rocking electrode vessel of about $125 \mathrm{cc}$. capacity was used, provided with connections similar to those of the Clark cell but with facilities for introducing a buret tip. The use of this apparatus avoided to a considerable extent errors due to change in carbon dioxide content during titration referred to by Tillman, ${ }^{8} \mathrm{John}-$ stor $^{9}$ and Kolthoff. ${ }^{10}$

In Fig. 1 the results of these titrations are shown graphically. In order to make the scale of the curves uniform the results have been so recalculated as to represent cubic centimeters of $0.1 \mathrm{~N}$ acid used per $100 \mathrm{cc}$. of solution titrated. The abscissa in the case of the $0.02 \mathrm{~N}$ sodium carbonate has twice the value that it has

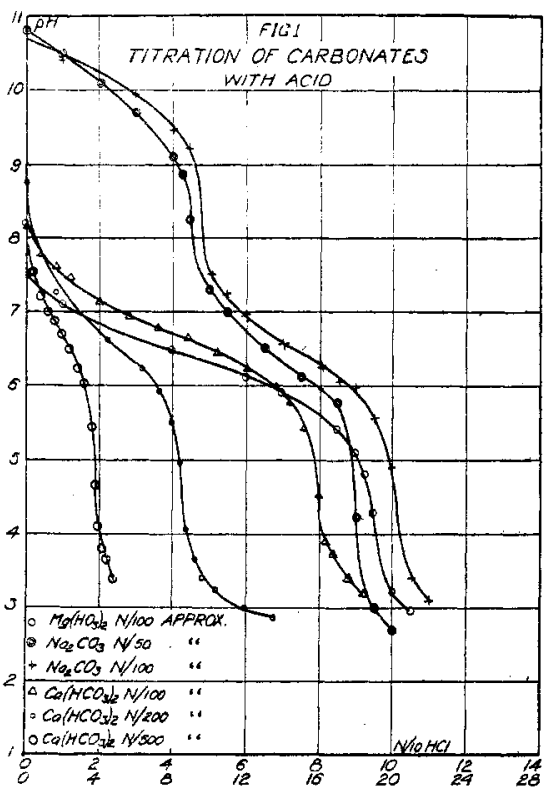
in all the other cases. The sodium carbonate solutions being normal carbonate show two inflection points while the others show only one.

It is to be noted that the position of the inflection point is not materially

\& Tillman, Stadt. Hyg. Univ. Inst. Frankfurt, M. Z. Nahr. Genussm., 38, 1 (1919).

Johnston, THIS Journal, 38, 947 (1916).

${ }^{10}$ Kolthoff, Chem. Weekbl., 14, 781 (1917); C. A., 11, 3454 (1917). 
affected by the nature of the metal ion. The concentration does affect the position of the inflection point somewhat, and this confirms the prediction to be made from a consideration of the mass-law equations involved.

This effect is not sufficient to necessitate the changing of the indicator used. If extreme accuracy is desired a color comparison standard corresponding to the particular Sörensen value desired could be used. It is also to be noted that the first, or carbonate, inflection point is about $P_{\mathrm{H}}$ 8.2. Since a solution of this value imparts only a faint color to phenolphthalein this indicator is seen to be satisfactory. (Any other indicator showing a good end-point color at this value would be equally satisfactory.) The second inflection point occurs at about $P_{H} 4.5$ for which methyl orange is an indicator. Since Acree ${ }^{11}$ has shown that the presence of sodium chloride, which is so often found in natural waters, tends to raise the hydrogen-ion concentration of this end-point it would not be disadvantagous to select an indicator showing its color change at a slightly lower Sörensen value. Since aluminum salts hydrolyze they will when present constitute a portion of the alkalinity. ${ }^{12}$ The use of an indicator changing at higher Sörensen value will tend to offset this error. This is a special case and one in which the most accurate results cannot be attained.

\section{The Precipitation of Magnesium as the Hydroxide}

The problem of removing magnesium from such a dilute solution divides itself into two parts. The first is that of determining when sufficient reagent, in this case always an hydroxide, has been added to throw as much of the magnesium out of solution as possible. The second is to so treat this precipitate as to cause it to coagulate and settle properly. We may hope to throw light on the first part by means of various chemical determinations and with the hydrogen electrode. The second part deals with the behavior of colloids and would require different methods of study.

11 Acree, Am. Chem. J., 36, 120 (1906).

${ }^{12}$ This point is to be fully discussed in a second paper now in preparation. It is, however, apparent that during the titration of a solution having an original Sorrensen value on the alkaline side of the methyl orange end-point and containing aluminum salts, some hydrion will have been used up in reversing the hydrolysis of the aluminum salts by the time the end-point $\left(P_{\mathrm{H}} 4.2\right)$ is reached. Fig. 4 may be made to represent such a titration by considering the units of alkali on the X-axis as negative quantities of acid. Suppose then that in this figure a line be dropped perpendicular to the $\mathrm{X}$-axis from a point on any one of the three curves representing the Sörensen value at the beginning of a titration, e. g., $P_{B} 5.5$ and a line be similarly drawn from a point on the same curve corresponding to $P_{\mathrm{B}} 4.2$, the distance intercepted on the axis between these two lines will represent the acid required to cause the change from $P_{\mathrm{H}} 5.5$ to $P_{\mathrm{B}} 4.2$. Since the "alkalinity" of a water is its acid capacity up to the methyl orange end-point it is seen that partially neutralized aluminum salts constitute a part of the alkalinity. 
Bartow and Lindgren ${ }^{13}$ have shown that in treating a magnesium solution with lime water the minimum content of magnesium is not reached until the alkalinity with phenolphthalein is somewhat greater than $1 / 2$ that with methyl orange, or until some free hydroxide is present in the solution. This would correspond to a Sörensen value slightly higher than that of a dilute normal carbonate solution or about $P_{\mathrm{F}} 11$. This approximate figure could also be deduced from a consideration of the solubility product of magnesium hydroxide and can be verified from a rather small scale titration curve published by Hildebrand. ${ }^{2}$

Due to the meagerness of the data available it was considered advisable to prepare titration curves using standard sodium hydroxide and magnesium salt solutions of various concentrations, and composition.

Since it was found that considerable time was required for the reaction to come to equilibrium after each addition of hydroxide the titration could not be carried on in a titrating electrode in the ordinary manner. Instead, various amounts of the hydroxide solution were added to a number of bottles containing the same amount of magnesium solution. The bottles were allowed to stand with frequent shaking for 24 hours or more, after which hydrogen-ion determinations were made on each. To prevent contamination with carbon dioxide from the atmosphere during addition of the chemical and withdrawal of sample, the bottles were fitted with stoppers carrying an arrangement of tubes similar to that of an ordinary wash bottle. When not in use the tubes were closed by means of rubber tubing and pinchcocks. Experiments were run using magnesium sulfate solutions in $0.02,0.01$ and 0.005 $N$ concentrations with sodium hydroxide, and magnesium bicarbonate 0.01 with lime water. In some experiments sodium chloride was added to the magnesium solutions until they were $0.1 \mathrm{~N}$ with respect to this salt. In Fig. 2 typical examples of all the experiments made are given

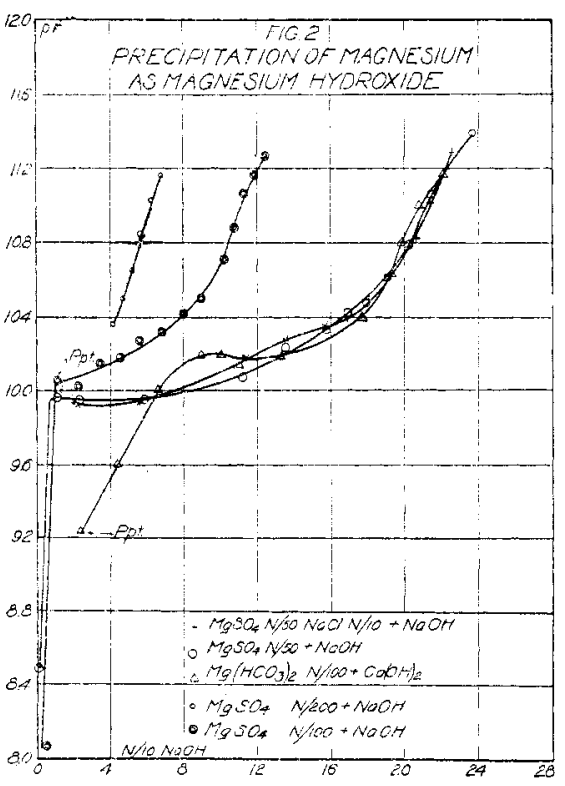
graphically. In this case the abscissas represent cc. of $0.1 \mathrm{~N}$ alkali added to $100 \mathrm{cc}$. of solution. These figures were obtained by calculation from the actual experimental values.

${ }^{13}$ Bartow and Lindgren, "Laboratory Experiments in Water Treatment," Illinois State Water Surv., Bull., 6, 33 (1908). 
The results show that the inflection point of the curve falls at practically the same hydrogen-ion concentration regardless of the concentration of the solution or the nature of the salt. This value is in the neighborhood of $P_{\mathrm{H}}$ 10.6. This value corresponds to a Sörensen value slightly lower than would be expected from consideration of the solubility product $1.2 \times 10^{-11}$. It is to be noted that Johnston ${ }^{14}$ considered that all values of the solubility product reported are too large. This deviation from the calculated value supports his opinion on this matter. The presence of sodium chloride in concentrations as high as $0.1 \mathrm{~N}$ does not materially affect the position or shape of the curve. It is especially noticeable that the curve for the titration of magnesium bicarbonate solution with lime water passes a maximum in the neighborhood of $P_{H} 10$. Since the reactions are somewhat complicated at this point it is difficult to assign an explanation. The solution is undoubtedly depositing both calcium carbonate and magnesium hydroxide. It is also probable that magnesium carbonate is being deposited. One explanation that might be offered is that one of the precipitates changes its physical state. That is, if one of the precipitates were colloidally dispersed it would probably have a solubility somewhat greater than the same substance would have in a non-colloidal state. If a certain hydroxyl-ion concentration should cause coagulation there might result a decrease in solubility and a corresponding decrease in the Sörensen value.

This explanation assumes that the coagulated precipitate is not peptized by the decrease in hydroxyl-ion concentration, which is not improbable as it is well known that these colloidal reactions are often not reversible in the strictest sense of the word. It is also true that while the formation of this maximum in the neighborhood of $P_{\mathrm{H}} 10$ is most noticeable in the case of the carbonate solution it is noticeable to some extent in most of the other experiments, but only to a very slight degree.

\section{Precipitation of Calcium Carbonate}

The precipitation of calcium carbonate is dependent first upon the concentration of the carbonate ion and in a somewhat secondary manner upon the hydrogen-ion concentration, since at higher hydrogen-ion concentrations the soluble calcium bicarbonate is formed. The subject of the solubility of this salt and the various factors influencing it has been very completely discussed by Johnston. ${ }^{15}$ He shows that the minimum solubility is about 16 parts per million and occurs at a partial pressure of carbon dioxide of about $3.73 \times 10^{-7}$ and an hydroxyl-ion concentration of $0.787 \times 10^{-4}$. This corresponds to about $P_{\mathrm{H}} 10$. If, however, the partial pressure of carbon dioxide is more nearly that of the atmosphere, the solubility is

14 Johnston, This Journal, 37, 2001 (1915).

is Johnston and Williamson, ibid., 38, 975 (1916). 
60 parts per million and the Sörensen value is in the neighborhood of $P_{\mathrm{H}} 8$.

Experiments were carried out on the precipitation of calcium from a calcium chloride solution using sodium carbonate and from a bicarbonate solution using lime water and the same methods as outlined for magnesium. Due to the high carbon dioxide pressure over these solutions it was difficult to get reproducible results. The results of three such runs are given graphically in Fig. 3. The results show that calcium carbonate begins to precipitate in solutions slightly more alkaline than $P_{\mathrm{H}}$ $i$ and that the inflection point of the curve is reached at about $P_{\mathrm{H}} 9.5$. This figure supports the calculated value of Johnston fairly well. Due to the extreme difficulty of sufficiently protecting these solutions from the carbon dioxide of the air

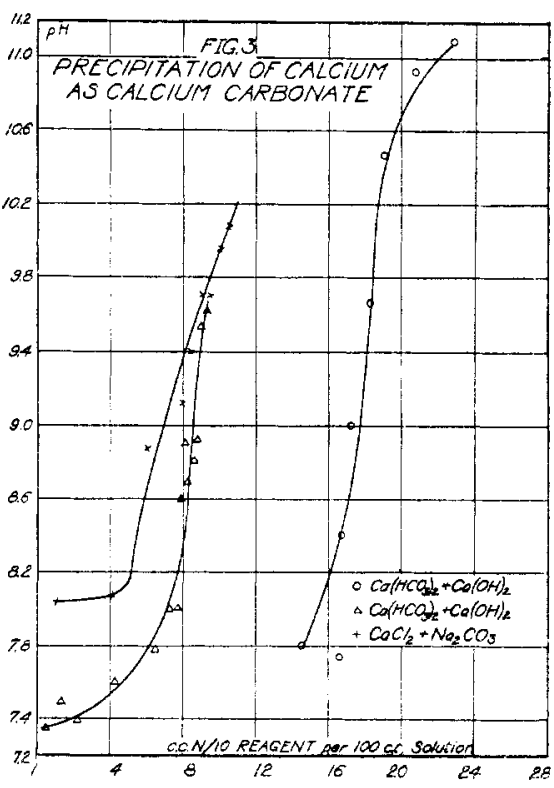
it is probable that the minimum solubility calculated by him was not attained. The difference in temperature between these experiments and those for which his results were calculated would render impossible too close comparison.

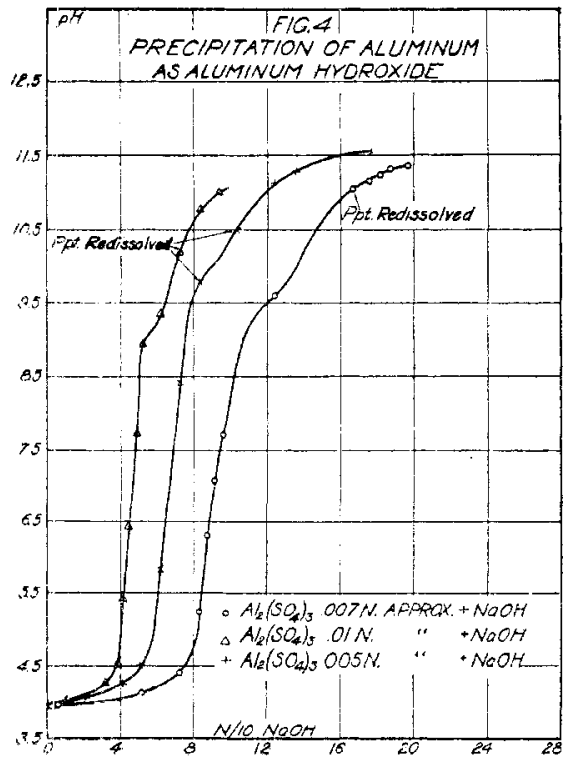

The Precipitation of Aluminum as the Hydroxide

The reaction between the natural bicarbonate alkalinity of water and aluminum sulfate is commonly used for the production of a coagulum in water to aid in the clarification and purification by either sedimentation or filtration. This precipitation of the aluminum as a hydroxide depends upon the hydrolysis of the bicarbonate to furnish the necessary hydroxyl ion. It has been shown by Hildebrand, Blum and others that aluminum hydroxide was precipitated to some extent at $P_{\mathrm{H}} 4$ and that it was precipitated most completely at from $P_{\mathrm{H}} 6.5$ to 7.5 and 
that the precipitate completely dissolved at a $P_{\mathrm{H}} 10$ to 11 . This resolution has been assigned to both a compound formation and to the peptization of the colloidal aluminum hydroxide. In an attempt to fix the zone of optimum precipitation somewhat more closely, experiments were run in a manner similar to those of magnesium. Solutions of aluminum sulfate approximately $0.01 \mathrm{~N}$ and $0.005 \mathrm{~N}$ were used. These results are given graphically in Fig. 4, where the abscissas are Sörensen values and the ordinates cubic centimeters of $0.1 \mathrm{~N}$ sodium hydroxide solution per $100 \mathrm{cc}$. of aluminum solution. As is to be seen from the results, in spite of the fact that a more dilute solution and a more sensitive apparatus were used, the optimum precipitation zone was not fixed within any narrower limits than previously. This reaction is being studied by other methods in this laboratory and further discussion of it will be deferred until the results of these studies are available.

\section{Summary}

Titration curves have been run with carbonates of sodium, magnesium and calcium, using a strong acid. It has been shown that the shape and position of the curve are unaffected by the metal ion, but that the inflection point occurs at a slightly higher Sörensen value in dilute solutions than in the more concentrated ones.

Precipitation curves of the precipitation of magnesium as the hydroxide were prepared. These showed that the precipitation of magnesium was complete at a Sörensen value in the neighborhood of $P_{\mathrm{H}} 10.6$. Magnesium hydroxide did not form until the value was as high as $P_{\mathbf{H}} 9.0$.

Precipitation curves of the precipitation of calcium as the carbonate while not as regular as those obtained in the precipitation of magnesium tend to show that the reaction is complete, sufficient carbonate being present at $P_{\mathrm{H}} 9.5$.

Aluminum hydroxide was shown to start precipitating in solutions as acid as $P_{\mathrm{H}} 4$ and to be completely precipitated at $P_{\mathrm{H}} 6.5$ to 7.5 . At values much higher than this re-solution commenced to take place; resolution was complete at a value between $P_{\mathrm{H}} 10$ and 11 .

The precipitation reactions were not instantaneous but took several hours to reach completion. This was more noticeable the more dilute the solution.

The precipitations of magnesium hydroxide and of aluminum hydroxide are being studied further in this laboratory.

URBANA, ILIINOIS 